\title{
Validation of the stream function method used for reconstruction of experimental ionospheric convection patterns
}

\author{
P. L. Israelevich ${ }^{1}$, V. O. Papitashvili ${ }^{2}$, A. I. Ershkovich ${ }^{3}$ \\ ${ }^{1}$ Department of Geophysics and Planetary Sciences, Raymond and Beverly Sackler Faculty of Exact Sciences, \\ Tel Aviv University, Ramat Aviv, Israel \\ e-mail: peter@jupiter1.tau.ac.il \\ ${ }^{2}$ Space Physics Research Laboratory, University of Michigan, Ann Arbor, Michigan, USA \\ (Also at Danish Meteorological Institute, Copenhagen, Denmark) \\ ${ }^{3}$ Department of Geophysics and Planetary Sciences, Raymond and Beverly Sackler Faculty of Exact Sciences, \\ Tel Aviv University, Ramat Aviv, Israel
}

Received: 28 June 1999 / Revised: 25 October 1999 / Accepted: 8 December 1999

\begin{abstract}
In this study we test a stream function method suggested by Israelevich and Ershkovich for instantaneous reconstruction of global, high-latitude ionospheric convection patterns from a limited set of experimental observations, namely, from the electric field or ion drift velocity vector measurements taken along two polar satellite orbits only. These two satellite passes subdivide the polar cap into several adjacent areas. Measured electric fields or ion drifts can be considered as boundary conditions (together with the zero electric potential condition at the low-latitude boundary) for those areas, and the entire ionospheric convection pattern can be reconstructed as a solution of the boundary value problem for the stream function without any preliminary information on ionospheric conductivities. In order to validate the stream function method, we utilized the IZMIRAN electrodynamic model (IZMEM) recently calibrated by the DMSP ionospheric electrostatic potential observations. For the sake of simplicity, we took the modeled electric fields along the noon-midnight and dawn-dusk meridians as the boundary conditions. Then, the solution(s) of the boundary value problem (i.e., a reconstructed potential distribution over the entire polar region) is compared with the original IZMEM/DMSP electric potential distribution(s), as well as with the various cross cuts of the polar cap. It is found that reconstructed convection patterns are in good agreement with the original modeled patterns in both the northern and southern polar caps. The analysis is carried out for the winter and summer conditions, as well as for a number of configurations of the interplanetary magnetic field.
\end{abstract}

Key words: Ionosphere (electric fields and currents; plasma convection; modeling and forecasting)

Correspondence to: P. L. Israelevich

\section{Introduction}

The polar ionosphere is electrically coupled with various magnetospheric domains by means of the geomagnetic field lines and, therefore, the ionospheric plasma convection can generally be considered as "a footprint" of the electric fields and currents flowing through the entire volume of the Earth's magnetosphere. Various papers can be found in the scientific literature where the polar cap ionospheric convection patterns are constructed from a vast amount of data: satellite measurements of the electric fields or ion drift velocities (e.g., Heppner and Maynard, 1987; Rich and Hairston, 1994; Weimer, 1995), polar cap ground magnetometer data (Feldstein et al., 1984; Richmond and Kamide, 1988; Papitashvili et al., 1994), and ground radar observations (Foster, 1983; Ruohoniemi and Greenwald, 1996), to name just a few.

A new approach for reconstruction of ionospheric convection patterns has recently been proposed by Israelevich and Ershkovich (1999) and this will be referred to herein as paper I. A polar satellite, which measures electric fields or ion drifts along the orbit, cuts across the entire polar cap and, therefore, both the electric potential along the track as well as the crosstrack potential derivatives become available. It is also reasonable to assume that the ionospheric electric potential is zero at lower, subauroral latitudes; hence, the polar region is surrounded by a boundary at which both the electric potential $\Phi$ and its normal (to the boundary) derivative $\partial \Phi / \partial n$ are equal to zero. Thus, if there is only a single satellite pass, the polar cap will be split in two areas for which the boundary conditions for electric potential and its normal derivative are known. As the number of polar passes increases, we obtain a number of polar cap areas with the known boundary conditions. Then, the ionospheric convection pattern can easily be found as a solution of the appropriate boundary value problem.

The proposed method is based on a few assumptions valid for the altitudes of $\sim 200-1000 \mathrm{~km}$. First of all, we 
neglect the curvature of the ionosphere assuming that it is a horizontal plane. Second, the main geomagnetic $\mathbf{B}_{0}$ is assumed to be constant over the polar cap and the field is parallel to a vertical axis $Z$ of the accepted coordinate system. Third, we assume that the fieldaligned currents close at lower altitudes of $\sim 100 \mathrm{~km}$. Under these assumptions $\partial / \partial z=0$ at the altitudes of several hundred kilometers and the boundary value problem becomes two-dimensional. We also assume that the magnetic field lines are equipotential and the magnetic field perturbations caused by these currents are small enough $\Delta B \ll B_{0}$.

It is known that the ionospheric plasma flow is incompressible. Indeed, a change of the total (that is, gaskinetic and magnetic) pressure in the steady-state flow is $\delta P=\delta\left(p+B^{2} / 8 \pi\right) \sim \rho_{0} v_{0}^{2}$, where $\rho_{0}$ and $v_{0}$ are the characteristic density and velocity in the flow, respectively; and $\delta p=(\partial p / \partial \rho)_{s} \delta p=c_{s}^{2} \delta p$, where $c_{s}$ is the sound velocity. On the other hand, $\delta B=B_{0} \delta \rho / \rho_{0}$ because of the "frozen-in" conditions for the magnetic fields and, therefore, $\left(\mathbf{B}_{0} \nabla\right) \mathbf{v}=0$. Thus,

$\delta P=\delta p+B_{0} \delta B / 4 \pi=c_{s}^{2} \delta \rho+V_{A}^{2} \delta \rho \sim \rho_{0} v_{0}^{2}$

and the flow is incompressible $\left(\delta \rho / \rho_{0} \ll 1\right)$ if $c_{s}^{2}+V_{A}^{2} \gg v_{0}^{2}$. The latter condition is always true in the polar ionosphere because the ionospheric convection velocity is much smaller than the Alfven velocity $V_{A}$. (Note that if the plasma flows along the magnetic field, the condition of plasma incompressibility is the same as in hydrodynamics: $c_{s}^{2} \gg v_{0}^{2}$.)

The Lorentz body force $\mathbf{j} \times \mathbf{B}=0$ because electric currents at these altitudes are field-aligned. Hence, a magnetohydrodynamic flow reduces to a hydrodynamic one, and it obeys the following equation (e.g., Landau and Lifshits, 1959):

$\frac{\partial}{\partial t} \nabla^{2} \Psi-\frac{\partial \Psi}{\partial x} \frac{\partial}{\partial y} \nabla^{2} \Psi+\frac{\partial \Psi}{\partial y} \frac{\partial}{\partial x} \nabla^{2} \Psi=0$

where $\Psi$ is a stream function $\left(v_{x}=\partial \Psi / \partial y\right.$; $\left.v_{y}=-\partial \Psi / \partial x\right)$.

It should be noted here that the hydrodynamic description Eq. (1) does not mean that the magnetic field is neglected. The magnetic field plays here an important role because (a) its strength is such that the 2-D flow is incompressible, and (b) it controls the relationship between the electric potential $\Phi$ and the stream function $\Psi$ in a steady state: $\Psi= \pm \frac{c}{B_{0}} \Phi$ (e.g., see paper I). Thus, for the steady state $\partial / \partial t=0$, the electric potential $\Phi$ satisfies the following equation:

$$
-\frac{\partial \Phi}{\partial x} \frac{\partial}{\partial y} \nabla^{2} \Phi+\frac{\partial \Phi}{\partial y} \frac{\partial}{\partial x} \nabla^{2} \Phi=0 .
$$

If the electric field measurements are available from one or more satellites, one can subdivide the polar cap into several areas whose boundaries are the low-latitude boundary at which $\Phi=0$ and $\partial \Phi / \partial n=0$ and the satellite tracks. Two components of the electric field vector measured by a satellite also provide $\Phi$ and $\partial \Phi / \partial n$ along the satellite trajectory. Therefore, the polar cap is divided into several parts (two parts if only one satellite orbit is available) with the boundary conditions for $\Phi$ and $\partial \Phi / \partial n$.

The problem of reconstructing the ionospheric convection pattern becomes a boundary value problem for Eq. (2). The main obstacle here is the fact that the solution of Eq. (2) with the known boundary conditions for $\Phi$ and $\partial \Phi / \partial n$ is not unique. However, among a variety of solutions, a single solution exists, which satisfies the following condition: every point inside the area is connected with the area boundaries by means of the stream lines. In other words, the latter condition means that there should be no closed-contour stream lines which are confined entirely within the selected area. Following this condition, we assume that every stream line (existing within the area) crosses at least one of the area boundaries (i.e., the low-latitude boundary or one of the satellite tracks. Therefore, the ionospheric convection pattern in the polar cap can be successfully reconstructed if the electric field (or ion drift) vector data are available along the satellite trajectories (i.e., boundaries) which cross all (or most) of the stream lines.

One of the ways to find the specific solution and to calculate the electric potential distributions over the polar cap is to find a steady-state solution of Eq. (1) using the relaxation method (see paper I). This solution would correspond to the realistic convection pattern if the available boundaries intercept all the stream lines; it would be much closer to the original, experimental distribution if most of the stream lines are intercepted. We have shown in paper I that it can hardly be satisfied by having a single satellite pass over the polar region; however, having two passes over the polar cap may produce quite satisfactory results when the trajectories divide the cap into approximately equal parts.

\section{Methodology}

The main goal of this study is a validation of the effectiveness of the stream function method for reconstruction of ionospheric convection patterns by using realistic distributions of the electric potential over high latitudes. It would be ideal to perform such a check calculating the potential distribution from the electric field vector measurements taken by two polar orbiting satellites, and then to compare the reconstructed pattern with simultaneous observations made by a third satellite. Unfortunately, we do not have three satellite observations at our disposal. Therefore, we have to utilize available experimental models of the ionospheric convection (i.e., electric potential distributions), for example, the potential patterns modeled by the IZMIRAN electrodynamic model (IZMEM, Papitashvili et al., 1994). This model provides experimental distributions of the electric potential for different orientations of the interplanetary magnetic field. In fact, these distributions have been obtained by analyzing a large amount of ground magnetometer data and then calibrated by the ionospheric electrostatic potential observations made under the Defense Meteorological Satellite Program (DMSP, Papitashvili et al., 1999). 
For the study, we utilized 2-D distributions of the electric potentials provided by the IZMEM/DMSP model (see details at http://www.sprl.umich.edu/MIST/). Then we calculated the potential distributions along two, perpendicular meridians (e.g., 12-00 MLT and 1806 MLT) and take these data as the boundary conditions (the IZMEM model assumes the zero potential boundary at $57^{\circ}$ of the corrected geomagnetic, (CGM, latitude). These meridians simulate the noon-midnight and dawn-dusk orbits of two polar satellites making near-simultaneous observations. Then we reconstructed the potential pattern as a solution of the boundary value problem and compared the obtained results with the original, experimental pattern taken from the model.

A description of the IZMEM model can be found elsewhere (e.g., Feldstein et al., 1984; Papitashvili et al., 1994, 1999). We took twenty IZMEM/DMSP potential distributions for the following IMF conditions: $B_{x}=0$, $B_{y}=0, B_{z}=-5 \mathrm{nT} ; B_{x}=0, B_{y}=0, B_{z}=0 ; B_{x}=0$, $B_{y}=5 \mathrm{nT}, B_{z}=0 ; B_{x}=0, B_{y}=0, B_{z}=5 \mathrm{nT} ;$ and $B_{x}=0, B_{y}=0, B_{z}=10 \mathrm{nT}$. We focused on the winter and summer distributions over both the northern and southern polar caps for each of these IMF situations. We specifically paid attention to the reconstruction of potential distributions for the northward IMF because of the possibility that the multi-cell pattern could be developed under that situation (e.g. Burke et al., 1979; Potemra et al., 1984; Reiff and Burch, 1985).

\section{Results}

Figure 1 (top) shows equipotentials for the IZMEM/ DMSP 2-D distribution of the electric potentials during winter conditions in the northern polar cap for the following IMF situation: $B_{x}=0, B_{y}=0$, and $B_{z}=-5 \mathrm{nT}$ (the contour interval is $5 \mathrm{kV}$ ). The figure shows a standard, two-cell convection pattern typical for the southward IMF conditions with a larger dawn cell. The boundary value problem is solved here in Cartesian coordinates related to polar geomagnetic coordinates as follows: $x=(90-L A T) \sin \left(M L T \cdot 15^{\circ}\right), \quad y=$ $-(90-L A T) \cos \left(M L T \cdot 15^{\circ}\right)$. The $(x, y)$ space consists of a uniform grid (33 by 33 points) over the region $-16<x<16,-16<y<16$. As the boundary conditions we chose the values of $\Phi$ and $\partial \Phi / \partial n$ along the axes $x=0$ and $y=0$. In this way we could simulate the electric field vector measurements made by the imaginary polar satellites flying along the dawn-dusk (18-06 MLT) and noon-midnight (12-00 MLT) meridians. These "satellite" tracks divide the polar cap into four areas: $-16<x<0,-16<y<0 ; 0<x<16$, $-16<y<0 ;-16<x<0,0<y<16$; and $0<x$ $<16,0<y<16$. In each of these areas we obtained the steady-state solutions for Eq. (1). Then the solutions were combined together to represent a reconstructed convection pattern shown at the bottom of Fig. 1. As one can see, the reconstructed electric potential pattern is in excellent agreement with the IZMEM/DMSP model potential distribution. The reconstructed pattern shows the same, two-cell convec-

\section{IZMEM model NWBZ-5}

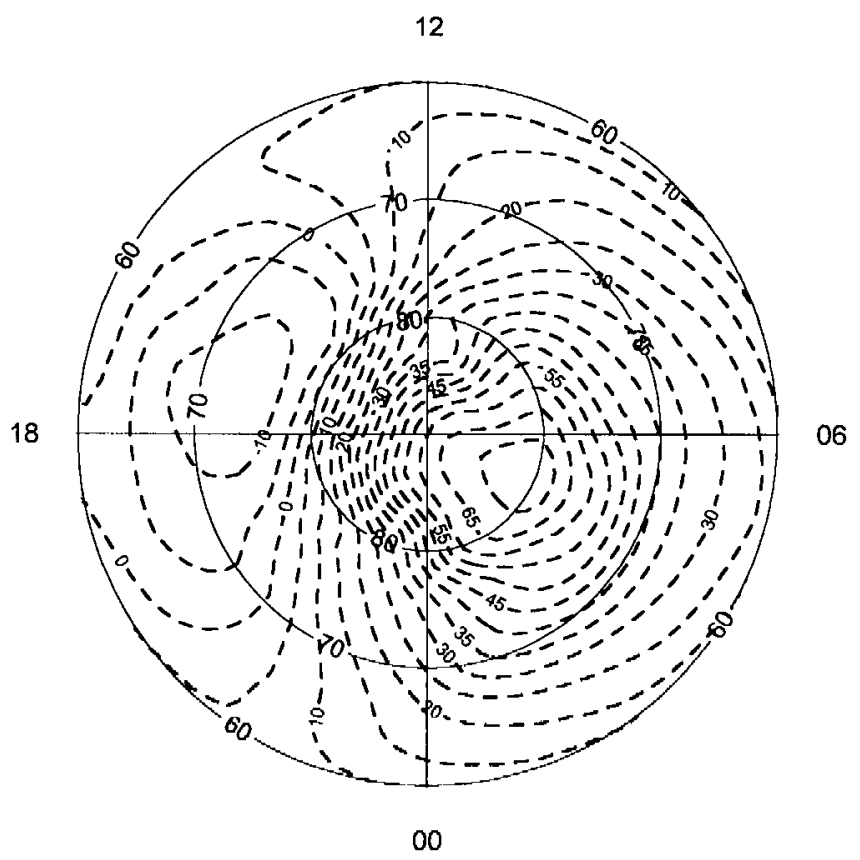

Reconstructed pattern

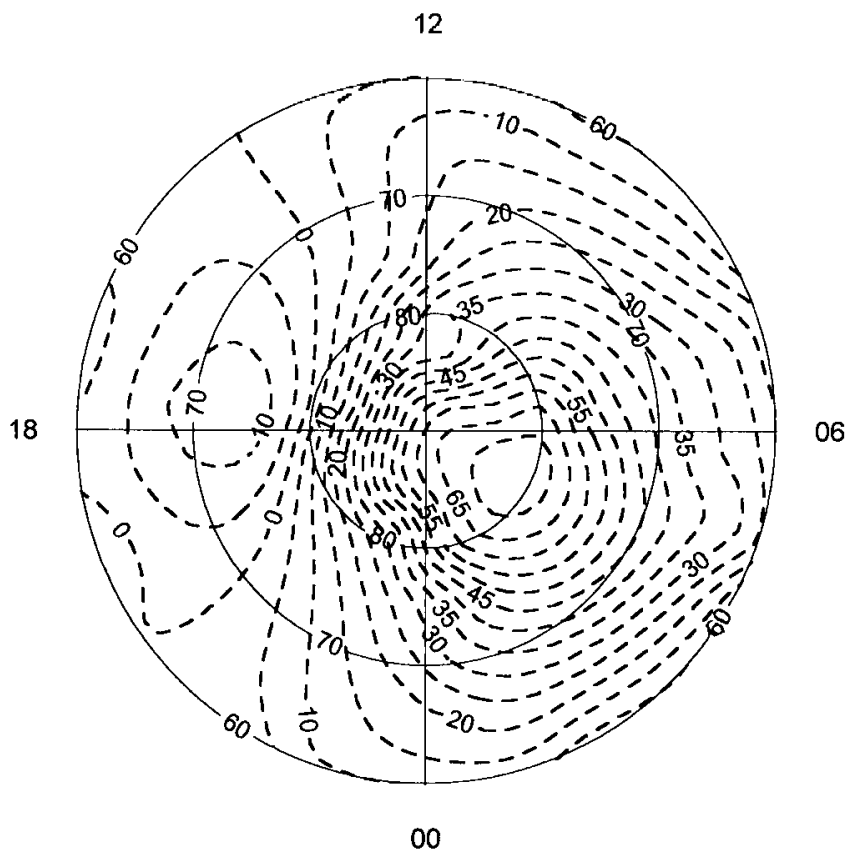

Fig. 1. Top distribution of the electric potentials in the northern winter polar cap for southward IMF $\left(B_{x}=0, B_{y}=0, B_{z}=-5 \mathrm{nT}\right)$ obtained from the IZMEM/DMSP model. Bottom distribution of the electric potentials reconstructed by the electric field data along the noon-midnight and dawn-dusk MLT meridians using the stream function method

tion pattern where location of the cells and the potential strength are very close to those shown by the original distribution. 
NWBZ-5
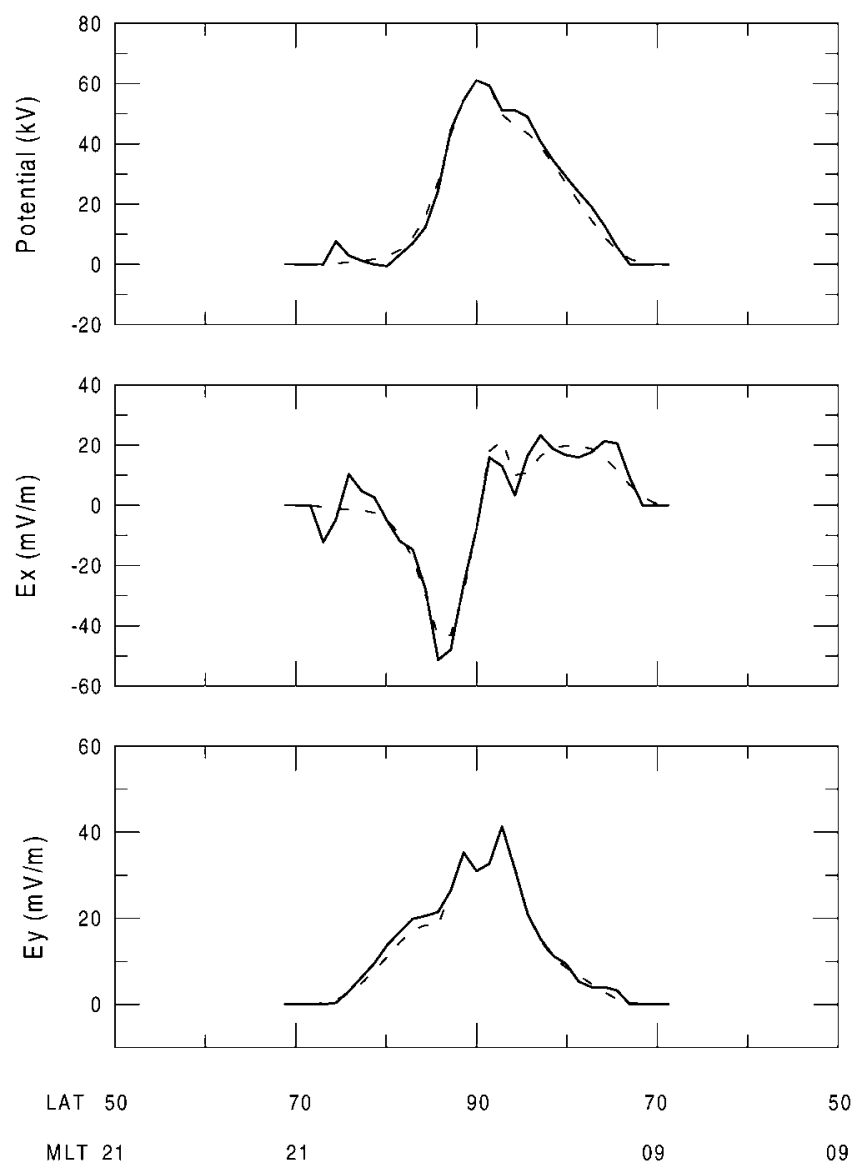

Fig. 2. Profiles of the electric potentials and electric field components $\left(E_{x}\right.$ and $\left.E_{y}\right)$ in the northern winter polar cap for IMF $B_{x}=0, B_{y}=0$, $B_{z}=-5 \mathrm{nT}$ along the MLT meridian 21-09. Solid lines are for the data from the IZMEM/DMSP model, dashed lines represent reconstructed values

The IZMEM/DMSP potentials and electric fields (solid lines) are compared with the calculated potentials and fields (dashed lines) along two other "satellite" tracks are compared in Figs. 2 and 3. These tracks simulate the orbits along the 21-09 MLT meridian (Fig. 2) and along the 15-03 MLT meridian (Fig. 3). One can see that the original and calculated potentials and fields are in good agreement. In order to compare the reconstructed and IZMEM/DMSP potentials over the entire polar region we calculated a correlation coefficient and made a linear fit to the data for all points of the grid (Fig. 4). As seen, the correlation coefficient between the reconstructed potentials and the IZMEM/ DMSP model is 0.99 .

The next few figures show results of the similar analysis for the potential distributions over the northern summer polar cap during the northward IMF condition $\left(B_{x}=0, B_{y}=0, B_{z}=5 \mathrm{nT}\right)$. The IZMEM/DMSP distribution of the electric potential in this case is shown in Fig. 5 (top). As seen, the standard, two-cell convection pattern is significantly distorted here; the dusk cell is larger than the dawn one, a few additional cells appear on the dayside $\left(\sim 12^{\mathrm{h}}, \sim 80^{\circ}\right)$ and near dusk
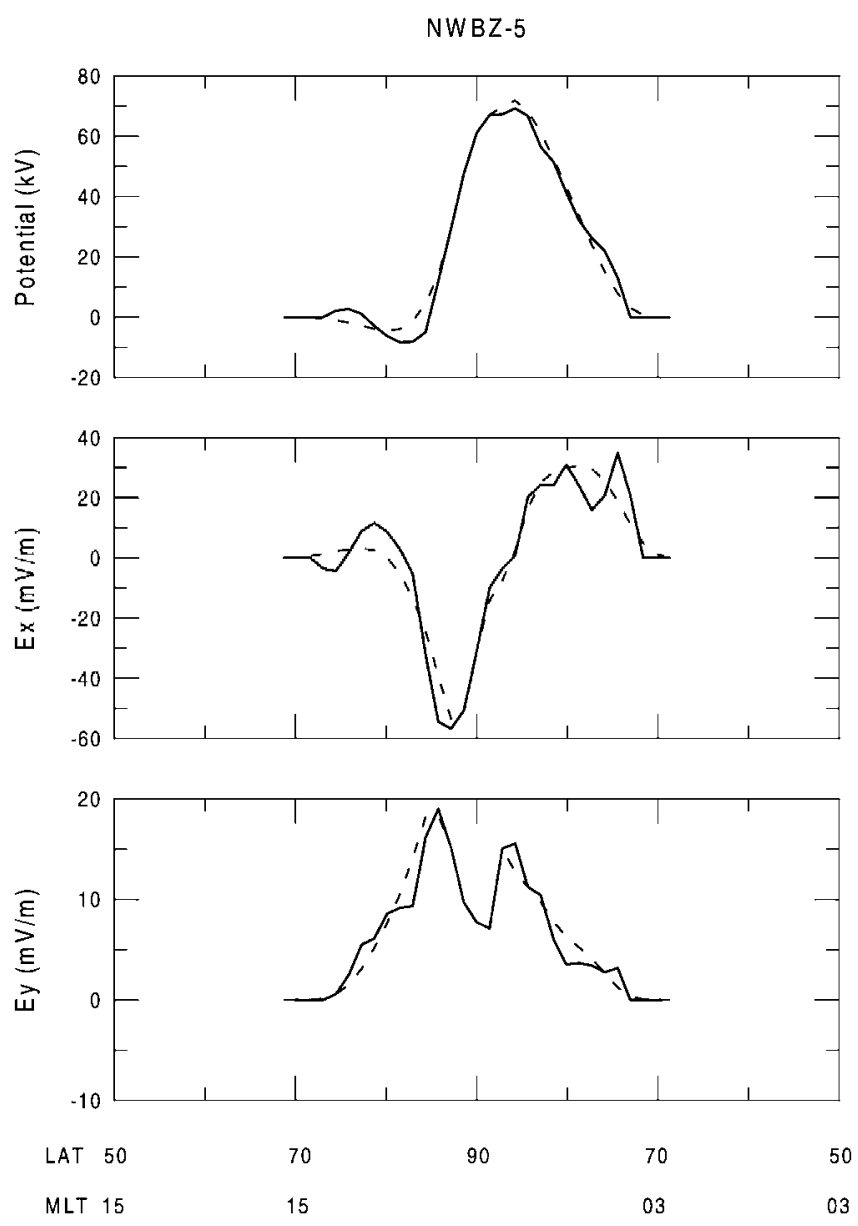

Fig. 3. Profiles of the electric potentials and electric field components $\left(E_{x}\right.$ and $\left.E_{y}\right)$ in the northern winter polar cap for IMF $B_{x}=0, B_{y}=0$, $B_{z}=-5 \mathrm{nT}$ along the MLT meridian 15-03. Solid lines are for the data from the IZMEM/DMSP model, dashed lines represent reconstructed values

$\left(\sim 19^{\mathrm{h}}, \sim 65^{\circ}\right)$. Here we choose the boundary conditions (that is, electric potentials and electric field components) along the 18-06 MLT and 00-12 MLT meridians. Note that this complicated convection pattern is also reconstructed rather well (bottom of Fig. 5). Here only the shape of low-latitude, dusk cell differs from those in the IZMEM/DMSP model. As a result, the convection in the MLT sector $15-18$ is poorly reproduced for latitudes lower than $70^{\circ}$.

The IZMEM/DMSP potentials and electric fields (solid lines) are compared in Figs. 6 and 7 with the calculated potentials and fields (dashed lines) for simulated satellite orbits along 21-09 MLT and 15-03 MLT meridians, respectively. The calculated potentials and fields reproduce those provided by the IZMEM/DMSP model reasonably well in this case. There is some difference between the IZMEM/DMSP and reconstructed distributions of the electric field $E_{x}$ component. In our reconstructed pattern some smaller scale variations of this component are smoothed. Nevertheless, the correspondence between the reconstructed and original patterns shown in Fig. 8 is very good. Here the calculated potential is plotted versus the model potential for all 
NWBZ-5

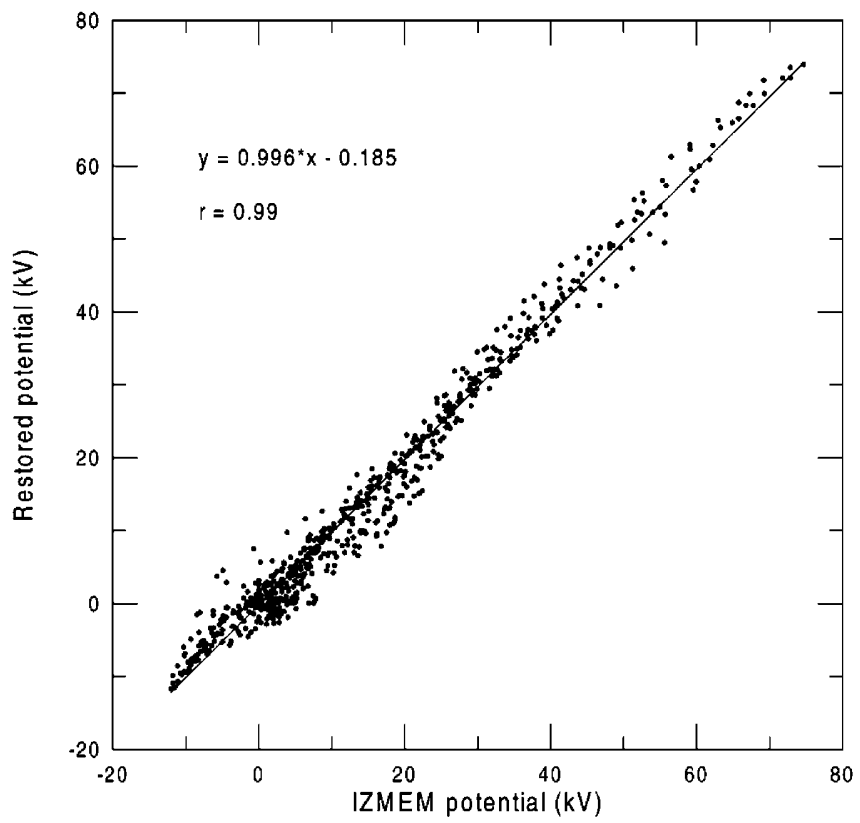

Fig. 4. Reconstructed electric potentials versus the IZMEM/DMSP potentials in the northern winter polar cap for southward IMF $\left(B_{x}=0, B_{y}=0, B_{z}=-5 \mathrm{nT}\right)$

points of the grid. Scattering of the points is somewhat larger than in the case of southward IMF (Fig. 4), but the correlation coefficient $r=0.96$ is still high.

These two examples of the convection pattern reconstruction are typical of the other 18 cases (not shown). The correlation coefficients between the electric potentials and its reconstructed values are close to unity in all cases. Figure 9 shows the reconstructed potential versus IZMEM potential for all points of the grid and for all twenty cases mentioned in Sect. 3: the correlation coefficient here is $r=0.98$.

\section{Concluding remarks}

The stream function method allows us to obtain twodimensional distributions of the electric potential over the polar region by using the very limited input data set, that is, electric field or plasma velocity vector measurements made along only two polar orbits. For the proprosed technique we do not need to know any information about ionospheric conductivities. The ionospheric convection over the polar cap ionosphere is described as a hydrodynamic, incompressible flow. This description is valid in the regions where the electric currents are field-aligned: $\mathbf{j} \times \mathbf{B}=0$. A unique solution of the hydrodynamic equation for the stream function exists for each area in the polar cap surrounded by the segments of satellite trajectories provided that the electric fields (or ion drifts), measured along these trajectories, are available. These measurements are taken as the boundary conditions; another assumption requires no closed contours of the stream lines within the bounded area. If the latter condition is satisfied, the
IZMEM model NSBZ5

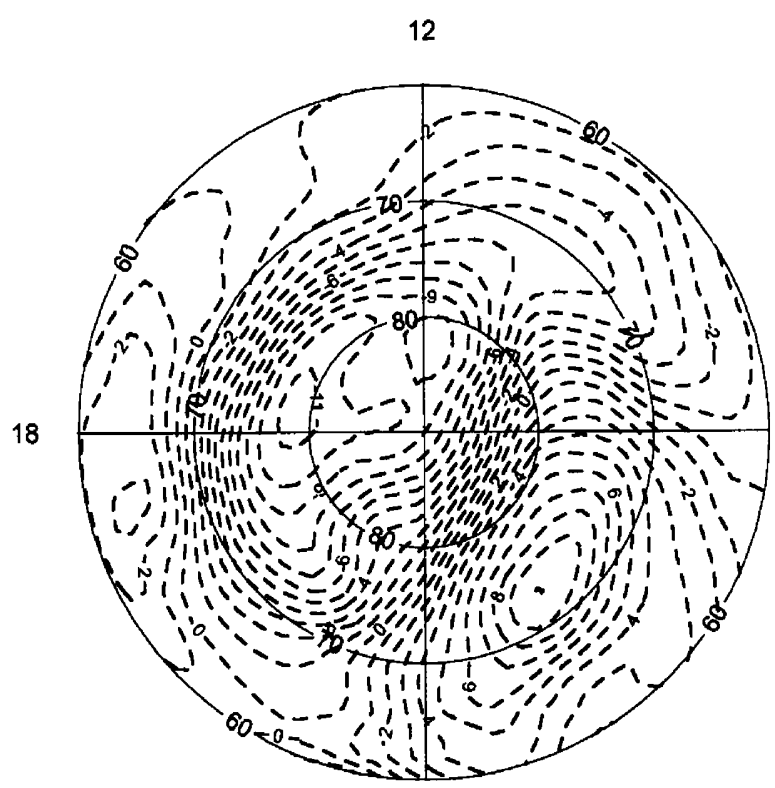

06

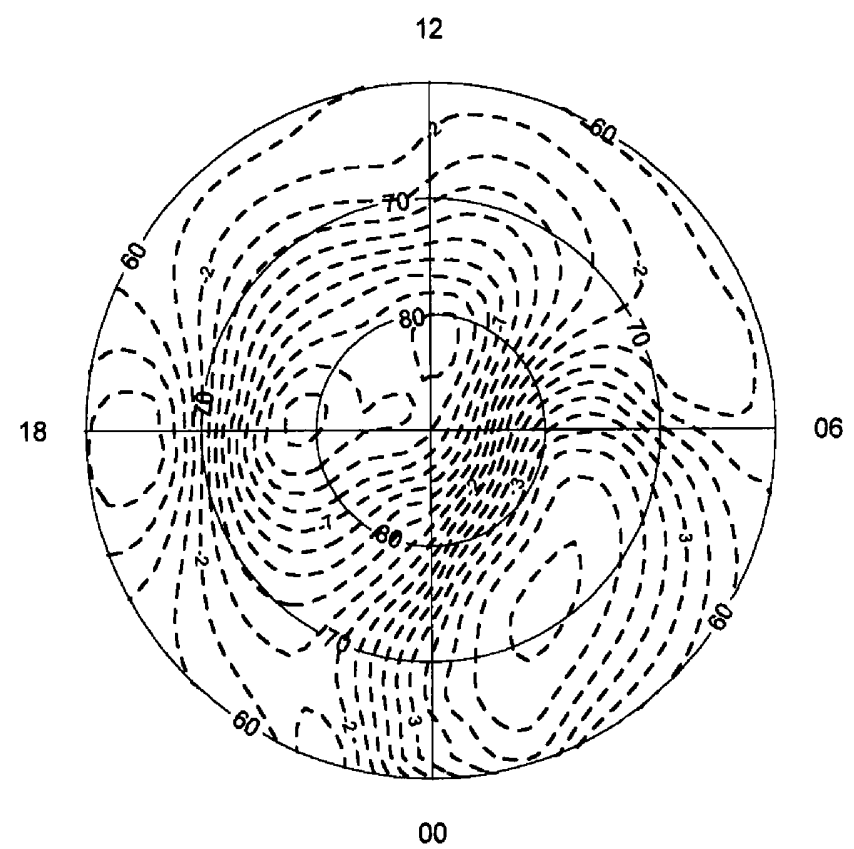

Fig. 5. Top distribution of the electric potentials in the northern summer polar cap for northward IMF $\left(B_{x}=0, B_{y}=0, B_{z}=5 \mathrm{nT}\right)$ obtained from the IZMEM/DMSP model. Bottom distribution of the electric potentials reconstructed by the electric field data along the noon-midnight and dawn-dusk MLT meridians using the stream function method

satellite trajectories cross all (or almost all) of the stream lines; this can be realised even if only two simultaneous satellite passes over the polar cap are available. It is hard to say in advance whether or not the orbits cross all the 
NSBZ5
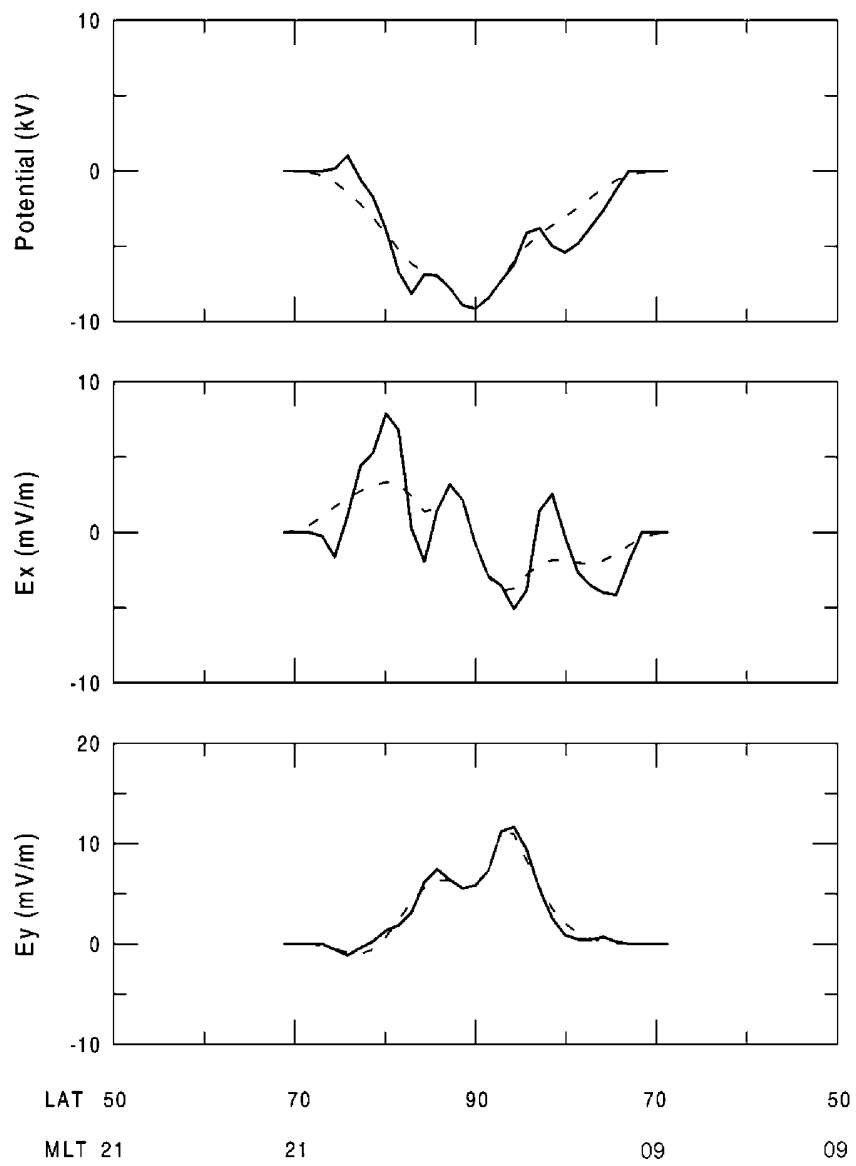

Fig. 6. Profiles of the electric potentials and electric field components $\left(E_{x}\right.$ and $\left.E_{y}\right)$ in the northern summer polar cap for IMF $B_{x}=0$, $B_{y}=0, B_{z}=5 \mathrm{nT}$ along the MLT meridian 21-09. Solid lines are for the data from the IZMEM/DMSP model, dashed lines represent reconstructed values

convection stream lines. Therefore, the practical criterion for the orbits choice should be as follows: the orbits are to divide the polar cap into regions with approximately equal area.

An ideal validation of the restored instantaneous convection pattern is to utilize some experimental data and then to check whether or not the restored pattern is similar to those obtained from an independent set of data obtained at the same time. In our case we would like to have had observations from two satellite orbits and then to compare the results with the measurements along a third orbit. Obviously, there are no appropriate data sets at present (we hope they will be available in the future). For this reason and in order to validate the stream line method, we took an experimental, statistical model and utilized only a limited set from the model data (i.e., "fake" satellite passes) for restoration of the entire pattern and then compared the restored pattern

Fig. 8. Reconstructed electric potentials versus the IZMEM/DMSP potentials in the northern summer polar cap for northward IMF $\left(B_{x}=0, B_{y}=0, B_{z}=5 \mathrm{nT}\right)$
NSBZ5
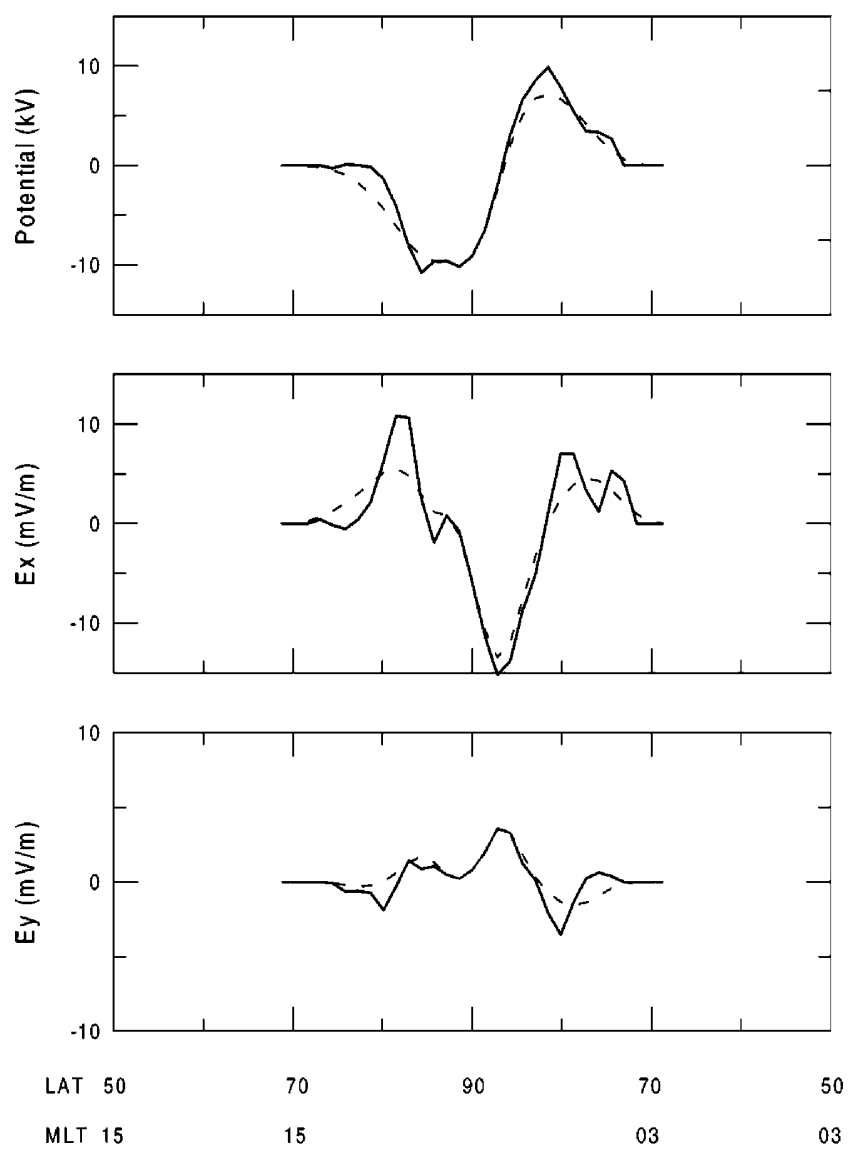

Fig. 7. Profiles of the electric potentials and electric field components $\left(E_{x}\right.$ and $\left.E_{y}\right)$ in the northern summer polar cap for IMF $B_{x}=0$, $B_{y}=0, B_{z}=5 \mathrm{nT}$ along the MLT meridian 15-03. Solid lines are for the data from the IZMEM/DMSP model, dashed lines represent reconstructed values

NSBZ5

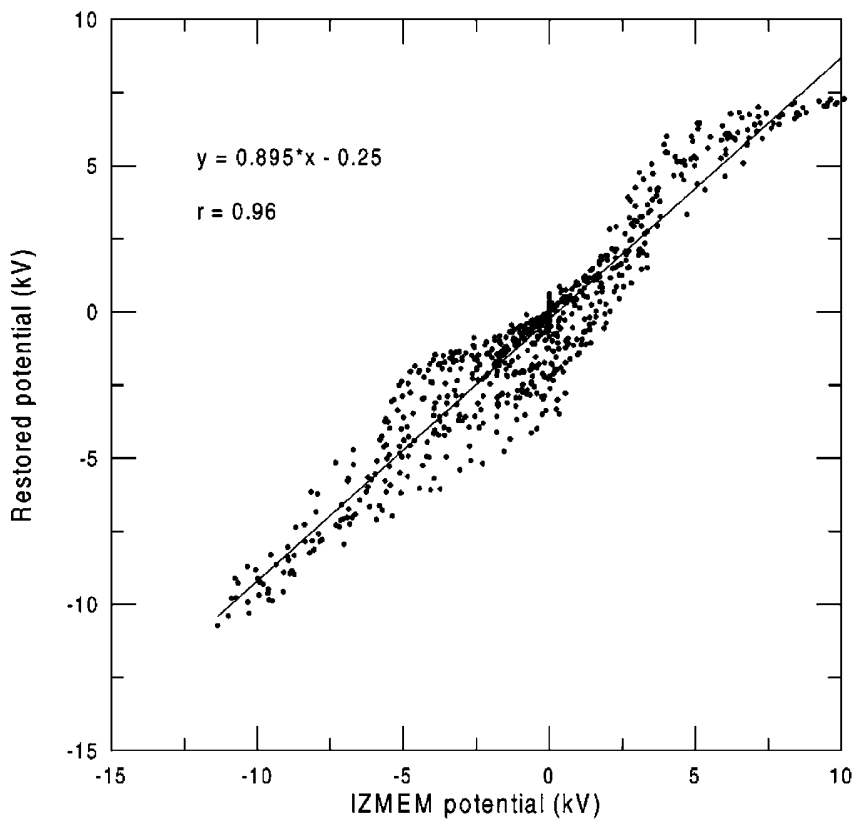




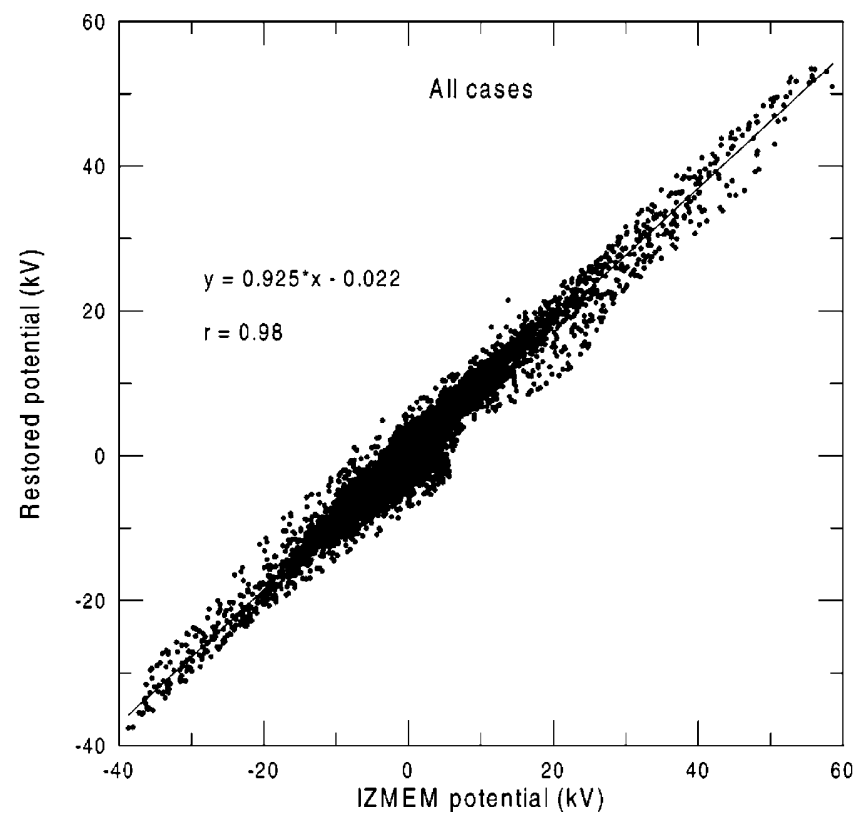

Fig. 9. Reconstructed electric potentials versus the IZMEM/DMSP potentials for all 20 cases

with the model one. An advantage of this approach is the fact that any experimental model always contains some noise, as a consequence of its presence in the input data used for the model construction. Hence, the stability of the stream line method with respect to the presence of the noise in the input is also verified.

We have validated the proposed new method using statistical convection patterns provided by the IZMEM/ DMSP model. Taking the electric field data along the noon-midnight and dawn-dusk meridians, we reconstructed the entire convection pattern for various orientations of the IMF. The reconstructed patterns appeared to be very close to the IZMEM-based distributions, thus proving the fact that real ionospheric convection is described by Eq. (2) and demonstrating the excellent abilities of the stream function approach.

Acknowledgements. V. O. P acknowledges partial support from the US National Science Foundation awards ATM-9523329, ATM-9614189, ATM-9628706 and ATM-9727554.

Topical Editor M. Lester thanks M. P. Freeman and F. Forme for their help in evaluating this paper.

\section{References}

Burke, W. J., M. C. Kelly, R. C. Sagalyn, M. Smiddy, and S. T. Lai, Polar cap electric field structure with a northward interplanetary magnetic field, Geophys. Res. Lett., 6, 21-24, 1979.

Feldstein, Y. I., A. E. Levitin, D. S. Faermark, R. G. Afonina, and B. A. Belov, Electric fields and potential patterns in the highlatitude ionosphere for different situations in interplanetary space, Planet. Space Sci., 32, 907-923, 1984.

Foster, J. C., An empirical electric field model derived from Chatanika radar data, J. Geophys. Res., 88, 981, 1983.

Heppner, J. P., and N. C. Maynard, Empirical high latitude electric field models, J. Geophys. Res., 92, 1115-1125, 1987.

Israelevich, P. L., and A. I. Ershkovich, A new method to reconstruct the ionospheric convection patterns in the polar cap, Ann. Geophysicae, 17, 743-749, 1999.

Landau, L. D., and E. M. Lifshits, Fluid mechanics, Pergamon, London, 1959.

Papitashvili, V. O., B. A. Belov, D. S. Faermark, Y. I. Feldstein, S. A. Golyshev, L. I. Gromova, and A. E. Levitin, Electric potential patterns in the northern and southern regions parametrized by the interplanetary magnetic field, J. Geophys. Res., 99, 13,251-13,262, 1994.

Papitashvili, V. O., F. J. Rich, M. A. Heinemann, and M. R. Hairston, Parametrization of the Defense Meteorological Satellite Program ionospheric electrostatic potentials by the interplanetary magnetic field strength and direction, J. Geophys. Res., 104, 177-184, 1999.

Potemra, T. A., L. J. Zanetti, P. F. Bythrow, A. T. Y. Lui, and T. Iijima, $B_{y}$-dependent convection patterns during northward interplanetary magnetic field, J. Geophys. Res., 89, 9753-9760, 1984.

Reiff, P. H., and J. L. Burch, IMF $B_{y}$ dependent plasma flow and Birkeland currents in the dayside magnetosphere 2. A global model for northward and southward IMF, J. Geophys. Res., 90, 1595-1609, 1985.

Rich, F. J., and M. Hairston, Large-scale convection patterns observed by DMSP, J. Geophys. Res., 99, 3827, 1994.

Richmond, A. D., and Y. Kamide, Mapping electrodynamic features of the high-latitude ionosphere from localized observations: technique, J. Geophys. Res., 93, 5741, 1988.

Ruohoniemi, J. M., and R. A. Greenwald, Statistical patterns of high-latitude convection obtained from Goose Bay HF radar observations, J. Geophys. Res., 101, 21,743, 1996.

Weimer, D. R., Models of high-latitude electric potentials derived with a least error fit of spherical harmonic coefficients, J. Geophys. Res., 100, 19,595, 1995. 